\title{
TRAYECTORIAS EDUCATIVO-LABORALES DE JÓVENES ESTUDIANTES DE EDUCACIÓN TÉCNICA EN CHILE: ¿TIENE SENTIDO UN SISTEMA DE FORMACIÓN PARA EL TRABAJO EN LA EDUCACIÓN SECUNDARIA?*
}

Educational-working paths among young students of technical education in Chile: Is it relevant to count on a training system for work at the secondary education level?

http://dx.doi.org/10.22235/pe.v9i2.1297

\section{LEANDRO SEPÚLVEDA V.*}

Recepción: 14-07-2016

Revisión: 09-09-2016

Aceptación: 12-09-2016

Resumen: El artículo analiza la trayectoria educativo-laboral de una cohorte de estudiantes egresados de la enseñanza media técnico-profesional en Chile. Los antecedentes permiten indicar que este sistema formativo ha dejado de ser una instancia de salida temprana al mundo del trabajo para un segmento relevante de jóvenes, quienes continúan estudios superiores en los años sucesivos a su egreso de la secundaria. Pese a esto, también se observa que un porcentaje cercano al $40 \%$ de los egresados no continúa estudios con posterioridad, y predominan experiencias laborales que tienden a estar mayormente disociadas del área formativa de los estudios cursados. La mayoría de estos jóvenes accede a empleos temporales, fundamentalmente en el sector servicios y en tareas que, en sentido estricto, no requerirían una preparación formal como la que se intenta desde el modelo curricular de la formación técnica. Esta dualidad señala la

\footnotetext{
* Este artículo se ha desarrollado en el marco del estudio "Modelos de trayectorias y desenlace educativolaboral de jóvenes: un estudio longitudinal en la Región Metropolitana" - Proyecto FONDECYT 1140596.

** Antropólogo social, doctor en Estudio de las Sociedades Latinoamericanas. Investigador del Centro de Investigación y Desarrollo de la Educación CIDE, Universidad Alberto Hurtado, Santiago de Chile.
} 
necesidad de una discusión profunda sobre el carácter de esta modalidad educativa, el lugar que tiene la formación de competencias para la empleabilidad en su interior y las reformas que eventualmente requerirá el sistema educativo en función de las experiencias de sus propios estudiantes.

Palabras clave: Sistema educativo; aspiraciones; estudiantes secundarios; transición educación-trabajo

\begin{abstract}
This article analyses educational-working paths of a cohort of students graduated from vocational high school in Chile. The background allows indicating that this training modality is no longer an instance of early exit to the world of work for an important segment of youngsters, who pursue higher education after high school graduation. However, it is also observed that approximately $40 \%$ of high school graduates do not pursue further studies, engaging in working activities that are not linked to the training received. Most of these students access to temporary jobs, mainly in the service sector and perform tasks that strictly would not require the formal training intended by the curriculum of technical education. This duality points out the need of a deep discussion about a number of issues regarding this educational modality: its characteristics, the place of employability skills training and the reforms eventually needed by the educational system based on the experiences of students themselves.
\end{abstract}

Keywords: Education system; aspirations; high school students; education-work transitions.

\title{
INTRODUCCIÓN
}

La formación para el trabajo y la educación técnica profesional han despertado un renovado interés en el debate educativo en los últimos años, y se ha observado un incremento de propuestas que enfatizan su importancia, tanto para el desarrollo productivo de los países como también para el fortalecimiento de procesos de inclusión social de los sectores más vulnerables de la población. En este marco, por ejemplo, UNESCO ha propuesto apoyar los esfuerzos que se realizan en los distintos países miembros de la organización. 
La estrategia aspira a apoyar los esfuerzos de los Estados Miembros para aumentar la pertinencia de sus sistemas de EFTP [enseñanza y formación técnica profesional] y dotar a todos los jóvenes y adultos con las competencias necesarias para el empleo, el trabajo decente, el espíritu empresarial y el aprendizaje a lo largo de toda la vida ${ }^{1}$ (UNESCO, 2016).

Aunque esta perspectiva discursiva ha sido relevada en otros momentos del debate sobre la estrategia de desarrollo que requeriría América Latina (por ejemplo, CEPAL, 1997), lo novedoso de la discusión actual es que, a diferencia de décadas pasadas, para el fortalecimiento de la educación técnica ${ }^{2}$ la escuela y el sistema escolar alcanzan una relevancia primordial. El significativo incremento de la matrícula de educación de nivel secundario y la ampliación de la oferta en el nivel de la educación superior (incluyendo el de carácter técnico), verificado en las últimas décadas en varios países de la región, constituyen, sin duda alguna, una base de sustento para el énfasis actual ${ }^{3}$. De igual manera, en el debate educativo, el impulso de nuevas propuestas curriculares orientadas a la implementación de modelos de profesionalización y el desarrollo de competencias de empleabilidad han incidido en la incorporación de un nuevo énfasis para la educación técnica, y ampliaron horizontes que, hasta hace poco, estaban mayormente remitidos al ámbito de la educación no formal (Maclean y Pavlova, 2013).

Como bien lo documentan Vera y Castioni (2010), en América Latina es posible observar dos grandes momentos de impulso de la formación para el

\footnotetext{
${ }^{1}$ Esta iniciativa se inserta en la Agenda 2030 para el desarrollo sostenible y enfatiza en: (a) fomentar el empleo y el espíritu empresarial de los jóvenes; (b) promover la equidad y la igualdad entre hombres y mujeres; (c) facilitar la transición hacia economías ecológicas y sociedades sostenibles.

${ }^{2}$ Aunque existen diversas formas de denominar a la modalidad, en función de la amplitud de campos formativos a los que se alude, se privilegia aquí la denominación genérica de educación técnica. El foco de interés de este trabajo está acotado al nivel de la enseñanza secundaria y terciaria, y los vínculos existentes entre estos sistemas en la actualidad.

${ }^{3}$ Con todo, es importante recalcar diferencias importantes de cobertura de matrícula y expansión del sistema de educación superior al comparar diversos países de la región. A esta heterogeneidad se agrega un cierto estancamiento del crecimiento de la cobertura en enseñanza secundaria, verificado en la última década en comparación con lo que ocurrió en el decenio anterior. Véase UNESCO, 2012.
} 
trabajo. El primero, asociado a las políticas desarrollistas y de industrialización que tuvieron lugar entre mediados del siglo XX y comienzos de la década del 70, y que depositaban en los Institutos Nacionales de Formación Profesional el desafío de preparar a la mano de obra calificada que demandaba la actividad productiva. El segundo, en cambio, corresponde a los desafíos de inserción de las economías en el mercado internacional y la apertura a la competitividad comercial en el marco de grandes transformaciones tecnológicas y restructuración de los mercados laborales. Es en este período donde se verifica, de manera mucho más evidente, la incorporación de la formación para el trabajo en la educación formal y el desarrollo de la educación técnica vocacional como un componente al interior del sistema educativo de los distintos países:

En el período se verificó un progresivo acercamiento de metodologías y estrategias entre ambos sistemas, especialmente en el caso de la educación media técnica. Se desdibujaron las fronteras entre la educación general, la formación profesional y el desarrollo tecnológico, y se impuso una necesaria interrelación, acorde al nuevo concepto de aprendizaje y formación a lo largo de la vida (Vera y Castioni, 2010, p. 8).

Por cierto, la realidad educativa actual sigue estando signada por este condicionamiento socioeconómico y por los preceptos que orientan las políticas educativas.

En la región, la educación técnica de nivel secundario presenta diferencias significativas tanto en relación a su cobertura como en sus modalidades. Existen varios países donde la interrelación entre el sistema formal de enseñanza y la institucionalidad de formación para el trabajo se encuentran mutuamente imbricados (Di Gropello, 2006). Lo anterior, incluso, puede llevar a cuestionar el levantamiento de juicios generales sobre este sistema formativo sin una referencia a los contextos institucionales específicos de cada país. Sin embargo, la gran heterogeneidad de los sistemas nacionales y la débil información disponible sobre muchos de estos dificulta los esfuerzos comparativos en perspectivas de una 
mirada de más largo alcance (Mejer, 2013). Con todo, la extensión en las últimas décadas de modelos en el nivel de educación secundaria, que validan la existencia de objetivos de desarrollo de competencias para el trabajo, y la posibilidad de continuar estudios post-egreso constituyen una tendencia relevante y motivan una serie de interrogantes investigativas hasta ahora poco exploradas.

En efecto, estas son algunas preguntas que pueden hacerse en relación a la actual provisión de la enseñanza técnica de nivel secundario: ¿Es pertinente, de acuerdo al actual escenario de expansión educativa, perseverar en la separación de un currículum académico tradicional respecto de uno propiamente vocacional en el nivel de enseñanza secundaria? ¿Cuánto incide la deselitización del sistema de educación superior en las trayectorias formativas de los jóvenes que participan de la educación vocacional? Al situar el foco en la educación secundaria, y el sistema educativo formal en general, el renovado énfasis en la discusión internacional sobre la importancia de la educación técnica, ¿representa un aporte a la equidad y mejores niveles de inclusión de los jóvenes más pobres 0 , por el contrario, refuerza un modelo de diferenciación que, en la práctica, puede perpetuar lógicas de segmentación social $?^{4}$

Por lo general, el tratamiento de este y otros temas ha estado mayormente centrado en el análisis de los marcos institucionales del sistema educativo y laboral; el debate curricular y su adecuación a las exigencias externas o las características de la provisión educativa de los centros escolares, y se han minimizado los procesos efectivos de trayectorias que experimentan los estudiantes y egresados del sistema, así como, de un modo más concreto, las significaciones y el comportamiento particular de los sujetos a partir de sus propias experiencias, que se verifican a lo largo del curso de sus vidas (Heinz, 2009).

En este artículo se sostendrá que el análisis de la experiencia y trayectoria educativo-laboral de los estudiantes que cursan esta modalidad formativa

\footnotetext{
${ }^{4}$ Por cierto, este no es un debate reciente. Hace casi un siglo John Dewey criticaba de manera consistente la existencia de dos modelos formativos y su efecto negativo en la reproducción de las desigualdades en la sociedad norteamericana (Stern, 2009). En la actualidad, la discusión también se verifica en los países pobres del sur, donde existen cuestionamientos respecto al lugar de la formación vocacional y el rol de los actores públicos y privados a cargo de su implementación (Wallenborn y Heineman, 2009).
} 
constituye un tema de relevancia para la discusión de políticas educativas de mediano y largo plazo, toda vez que estas experiencias informan sobre aspectos medulares de la definición de políticas y los supuestos sobre los cuales estas son construidas. Considerar lo que ocurre con los sujetos y sus experiencias, durante y después de su paso por una experiencia formativa, es una tendencia investigativa en el campo de la educación que puede ser muy fructífera para el análisis de la educación técnica en la región. De esta tendencia, hasta ahora, existen solo aproximaciones fragmentadas en los países de Latinoamérica ${ }^{5}$.

Este artículo pretende ser un insumo para la discusión, al presentar algunos antecedentes de un estudio realizado en el contexto de la realidad chilena. La educación técnica de nivel secundario en Chile representa una modalidad formativa que, en las últimas tres décadas, ha tenido un rol relevante en canalizar la demanda por educación de los sectores más pobres de la sociedad. En los años recientes, las nuevas generaciones de egresados de este sistema experimentan procesos complejos de transición. Se enfrentan al dilema de la inserción temprana en el mundo del trabajo y/o la continuidad de estudios, debido a la amplia oferta del mercado educativo de nivel superior en el país y las propias condiciones que ofrece el mercado de trabajo. A partir de la revisión de la trayectoria de una cohorte de egresados de este sistema formativo, se da cuenta de las condiciones educativo-laborales que prevalecen en este tiempo y, en función de los antecedentes disponibles, se discute acerca de la pertinencia de un sistema formativo de educación técnica a nivel de la enseñanza secundaria.

\section{EDUCACIÓN TÉCNICO PROFESIONAL DE NIVEL SECUNDARIO EN CHILE: ANTECEDENTES GENERALES}

La Educación Media Técnico Profesional (EMTP) representa una modalidad formativa de amplia cobertura en la enseñanza secundaria en Chile. El total de

\footnotetext{
${ }^{5}$ Pueden destacarse los trabajos de Jacinto (2010), Miranda y Otero (2005), Fridman y Otero (2015) en Argentina, y Dávila, Ghiardo y Medrano (2005) en Chile.
} 
estudiantes bajo este sistema supera los 180.000 jóvenes, lo que representa el $40 \%$ del total de la matrícula de los niveles de $3^{\circ}$ y $4^{\circ}$ año de enseñanza media ${ }^{6}$. La gran mayoría de la población escolar corresponde a estudiantes que provienen de familias de los sectores de menores ingresos de la sociedad: el $65 \%$ de los jóvenes de los dos deciles más pobres que estudia enseñanza media lo hace en este sistema. Aunque no existe información consistente acerca de las variables más relevantes que inciden en la actual opción de estudios al interior de la EMTP, investigaciones recientes coinciden en destacar que el nivel socio-económico opera como el factor más determinante, por encima del desempeño escolar alcanzado en la educación básica, y plantean la interrogante acerca del carácter reproductor de desigualdad que puede implicar la existencia de este sistema formativo (Larrañaga, Cabezas y Dusaillant, 2013; Farías y Carrasco, 2012).

La actual oferta de la EMTP en Chile surgió de una reforma educacional, implementada durante la década de los noventa, que tenía como objetivo central mejorar la calidad y la equidad de la educación, al favorecer el desarrollo de contextos de aprendizaje funcionales a las demandas sociales y generar oportunidades de aprendizaje que permitiesen alcanzar mayores niveles de inclusión y permanencia de los jóvenes en el sistema escolar. A comienzos de esta década, tanto en el país como en el resto de la región, se resaltaban las dificultades para dar respuesta a los nuevos desafíos de desarrollo económico en el marco de las transformaciones productivas mundiales. Se diagnosticaba un marcado desajuste entre el sistema educacional tradicional y los nuevos requerimientos económicos y sociales, derivados de "un paradigma productivo que cambia vertiginosamente y que basa cada vez más su desarrollo en el conocimiento, el progreso técnico, la innovación y la creatividad" (CEPALUNESCO, 1992, p. 119).

\footnotetext{
${ }^{6}$ En el nivel de enseñanza secundaria, el sistema chileno reconoce dos modalidades formativas diferenciadas en los dos últimos años de educación obligatoria (11 y 12 grado): la educación científica humanista, que prioriza un modelo formativo conducente a estudios superiores, y la educación técnicoprofesional, que corresponde al foco de interés de este artículo.
} 
Desde entonces, la orientación de políticas curriculares y el apoyo al desarrollo de las propuestas formativas en los centros educacionales han estado guiados por el interés de desarrollar competencias para el trabajo en los jóvenes estudiantes. Se busca que esta formación entregue herramientas adecuadas para la incorporación al mundo laboral y, muy particularmente, para el enfrentamiento de los cambios tecnológicos que caracterizan el tiempo actual.

Curricularmente, la EMTP se organiza en 15 sectores económicos y 35 vías de especialización. El modelo formativo asume el enfoque de competencias laborales, y la forma de organización de la enseñanza es a través de la distribución modular de los programas de estudio. En las vigentes Bases Curriculares de la Formación Diferenciada Técnico-Profesional de la Educación Media se ha definido un contexto laboral y un conjunto de objetivos de aprendizaje para cada especialidad, que deben ser logrados al final de los dos años de formación diferenciada. Estos objetivos configuran el perfil de egreso de los estudiantes, al explicitar lo mínimo y fundamental que debe aprenderse en la especialidad. Esto incluye las competencias técnicas propias de la especialidad, o de la respectiva mención, así como también los objetivos de aprendizaje genéricos que son comunes a todas las especialidades y que remiten a competencias transversales independientes del sector económico de cada una de estas (Miranda, 2005; Sevilla, 2012). La definición curricular vigente se fundamenta en la existencia de alternativas educativo-laborales amplias para los egresados del sistema y en una perspectiva de trayectoria que inhibe su consideración como fase terminal del proceso formativo de los estudiantes.

El espacio de Formación Diferenciada, correspondiente a los niveles $3^{\circ}$ y $4^{\circ}$ de educación media, ofrece a los estudiantes oportunidades de realizar aprendizajes en una especialidad técnica que facilite su acceso a un primer trabajo remunerado, atendiendo a sus intereses, aptitudes y disposiciones vocacionales, y que los prepare en forma efectiva para el trabajo y para responder con flexibilidad a la velocidad de los cambios tecnológicos. Con igual 
Trayectorias educativo-laborales de jóvenes estudiantes de educación técnica en Chile importancia, el espacio de la Formación Diferenciada TécnicoProfesional considera también la continuidad de estudios técnicos como un destino posible y deseable de los egresados. En el diseño de los perfiles de egreso se ha considerado resguardar una perspectiva de itinerarios de formación técnica en un sistema de formación permanente. Así, la mayoría de las especialidades propuestas tiene continuidad en la oferta de formación de técnicos de nivel superior de los Centros de Formación Técnica, Institutos Profesionales y universidades del país (Ministerio de Educación, 2013, p. 11).

Más allá de estas definiciones curriculares, algunos estudios dan cuenta de varias falencias en este sistema formativo. Así, por ejemplo, Espinoza, Catillo y Traslaviña (2011) advierten sobre las dificultades de implementación de los módulos de formación de la EMTP debido a lo ambicioso de los contenidos curriculares de las especialidades vigentes, las limitaciones de tiempo para el cumplimiento de los objetivos curriculares y la carencia de recursos al interior de los establecimientos educacionales para un adecuado cumplimiento del trabajo formativo. Desde una mirada evaluativa global, el Reporte de la Comisión Externa de Formación Técnica para el Ministerio de Educación (2009) señala las grandes debilidades institucionales para alcanzar una formación de calidad, debido a la escasez de recursos materiales e insumos para el trabajo práctico en los establecimientos, las debilidades de formación de la gran mayoría de los docentes que trabajan en los establecimientos educacionales, así como la desvinculación que existe entre este modelo formativo, la oferta de educación técnica de nivel superior y el mundo productivo. Este diagnóstico de la EMTP continúa vigente hasta ahora (véase, por ejemplo, Educación 2020, 2016) pese a que no existen estudios con evidencia empírica relevante que se hayan desarrollado en el tiempo reciente.

Sepúlveda, Ugalde y Campos (2011), al estudiar la opinión de los actores educativos del sistema, destacan la ambigüedad estratégica de la EMTP 
impulsada por los agentes educativos, que, más allá de las definiciones de política, se caracteriza por el predominio de un modelo dirigido al ingreso laboral temprano de sus egresados y una débil vinculación entre la formación general y aquella propiamente de las especialidades técnicas. También es posible verificar este discurso en agentes productivos e, incluso, en responsables de políticas públicas. Esta indefinición de la estrategia formativa se debe, en gran parte, a la ausencia de soportes institucionales más consistentes, que otorguen proyección y perspectiva a esta modalidad de enseñanza. La expresión más evidente de lo anterior es la escasa o nula vinculación de la EMTP y sus instituciones con la oferta formativa post-secundaria que existe en el país. De este modo, aunque predomina un discurso transversal acerca de un objetivo múltiple y no terminal de esta modalidad formativa, internamente esta perspectiva no siempre tiene un correlato en las prácticas concretas, ya que existen lógicas de formación tradicional entre agentes educativos y orientadores vocacionales que puede provocar un efecto negativo en las aspiraciones y proyectos de futuro de sus estudiantes.

Pese a lo anterior, en los últimos años se observa un incremento de la participación de los egresados de la EMTP en la educación superior (y muy particularmente en la educación técnica terciaria), con la consecuente postergación de la incorporación al mundo laboral de un segmento relevante de jóvenes: en la actualidad cerca del $40 \%$ de los egresados de este sistema formativo continúa estudios post-secundarios al finalizar su enseñanza media (SIES-MINEDUC, 2014).

Los antecedentes que entregan estos estudios sobre la EMTP han planteado la necesidad de una discusión profunda sobre el carácter de esta modalidad formativa y las reformas que eventualmente requerirá (Sevilla, Farías y Sepúlveda, 2013). De igual manera, los importantes cambios en la situación postegreso de los estudiantes de este sistema interrogan acerca de la pertinencia de sostener una modalidad de formación vocacional a nivel medio (por lo menos de la magnitud observada en la actualidad en el país), teniendo en consideración el crecimiento sostenido de la matrícula de esta modalidad formativa en el nivel de la 
educación post-secundaria. Analizar la experiencia de egreso y la trayectoria educativo-laboral de los jóvenes que cursan en este sistema resulta, de este modo, un insumo necesario para la evaluación del modelo formativo y sus eventuales ajustes hacia el futuro.

\section{PERSPECTIVA ANALÍTICA}

La apertura al análisis de las estrategias activas de desarrollo personal, construcción de proyectos de vida y transiciones educativo-laborales representa una perspectiva emergente en la investigación, que ha alimentado diversos estudios sobre la incorporación masiva de jóvenes a la educación superior, la postergación del ingreso a una vida laboral plena, la ampliación temporal de la experiencia de combinación de estudio y trabajo, así como también la tendencia de inserción precaria al mercado laboral bajo las nuevas condiciones del orden económico internacional (Heinz y Krüger, 2001; Furlong, 2009).

El análisis de dichos fenómenos ha planteado la necesidad de estudiar los procesos de curso de vida (transición educativo-laboral o transición de la vida dependiente a la plena autonomía), considerando tanto los condicionamientos de la estructura social y el marco institucional en que estos se verifican, como la experiencia concreta que viven los sujetos (Evans, 2002; Machado Pais, 2000). Esta línea de investigación destaca la importancia de favorecer un enfoque que se centre en la relación sujeto-estructura, con un claro énfasis en una perspectiva que recupera a los individuos y su experiencia como núcleo relevante de la reflexión analítica (Woodman y Wyn, 2011).

Los estudios en el área (por ejemplo, Staff y Mortimer, 2003; Furlong, 2011) destacan la importancia de esta perspectiva en función de las grandes transformaciones ocurridas en las últimas décadas, que evidencian el debilitamiento de modelos de transición uniformes asociados a la condición de clase social o categoría de edad. Estas investigaciones recalcan que los actuales procesos de transición que experimentan las nuevas generaciones adquieren una dimensión mayormente individualizada, centrada en las expectativas, estrategias y 
capital específico que acumula el sujeto, por sobre las orientaciones colectivas de tipo transversal. En este marco, se verifica una pérdida de sentido de la transición como una secuencia normativa y lineal. El modelo tradicional de dependencia preparación para la vida adulta - adultez, que correspondía secuencialmente al paso de la vida escolar o estudiantil al ingreso al mundo del trabajo, se ha complejizado en una serie de otras variantes de transición, que incorporan un mayor nivel de complejidad e indefinición acerca de sus límites temporales y espaciales.

De igual modo, las actuales modalidades de transición que caracterizan al período juvenil se alargan temporalmente y posponen, para una gran masa de individuos, la asunción de roles tradicionales. El incremento de los años de escolaridad promedio, por una parte, y la postergación de las decisiones de formar familia o realizar compromisos que obliguen a la autonomía económica, por otra, son dos de los rasgos más sobresalientes asociados a este hecho (Leccardi y Ruspini, 2006). Con todo, algunos autores advierten que, pese a la heterogeneidad y diversidad de experiencias que es posible identificar en la investigación empírica, los factores estructurales siguen jugando un rol determinante en las posibilidades y alternativas de elección de los sujetos, por lo que la individualización del tiempo actual no debe confundirse con una simple biografía de elección, que ocultaría o ayudaría a enmascarar las estructuras de desventaja que operan en la realidad social (Biggart, Furlong y Cartmel, 2008; Cieslik y Simson, 2013).

La importancia de este enfoque radica en que las políticas en general, y las de educación y trabajo en particular, deben ser analizadas y evaluadas en función de la experiencia que viven los sujetos hacia los cuales están dirigidas. Sarojini (2014), recogiendo el aporte de Stephen Ball en el campo educativo, señala que, a diferencia de una perspectiva lineal, resulta más adecuado considerar a la política como un sistema cíclico, que es creado y recreado a lo largo del tiempo por los distintos actores que participan o inciden en este. En otras palabras, la política no se agotaría en su momento legislativo, ya que contiene una carga importante de 
intertextualidad donde las aspiraciones y conductas subsecuentes de los individuos adquieren una gran relevancia”.

Bajo esta perspectiva, Wyn y Dwyer (2000) señalaron, tempranamente, que existen dos temas principales asociados a este proceso, que tiene una directa vinculación con el debate acerca del sistema educativo: (a) el reconocimiento de que existe una dificultad para adecuar una propuesta de política educativa a los cambios fundamentales experimentados en el sistema productivo y la organización de la sociedad. Desde este punto de vista, el sistema escolar, en términos generales, parece seguir siendo construido sobre la base de un modelo de transición predefinida, respondiendo escasamente a los nuevos desafíos de integración social del modelo productivo predominante; (b) junto a esto, se destaca el giro de expectativas, significaciones y propuestas de futuro que expresan las nuevas generaciones, en contradicción con las perspectivas de desarrollo profesional que predominan en el modelo escolar tradicional. Particularmente los jóvenes de sectores socialmente en desventaja reconocen el conjunto de obstáculos y limitaciones que ofrecen las tradicionales perspectivas profesionales, lo que los obliga a replantearse prioridades y expectativas, tanto en el ámbito de un proyecto profesional-laboral como en todas las dimensiones de la organización de la vida cotidiana.

Para estos autores, el reconocimiento de las profundas transformaciones de la llamada era post-industrial pone en cuestión la viabilidad y asunción pasiva de un modelo predefinido de tránsito a la edad adulta normal de los jóvenes.

No podemos dar por sentado el programa predefinido (que aún influye en la actual generación de responsables de las políticas de educación y de investigadores de la juventud). Tenemos que someterlo a prueba, contrastándolo con los programas de los

\footnotetext{
${ }^{7}$ En una línea similar, Willis (2003) señala la importancia de reconocer las respuestas sociales (bottom-up responses) a las prácticas institucionales (top-down practices), que buscan organizar el orden a partir de la definición de políticas. Los nuevos escenarios de modernización, para este autor, corresponden a un campo específico de interacción de ambos procesos y una lectura compleja de la realidad social obliga a una mirada que incluya tales interacciones.
} 
Trayectorias educativo-laborales de jóvenes estudiantes de educación técnica en Chile

propios jóvenes como respuesta a las diferentes condiciones vitales de la era post-industrial (Wyn y Dwyer, 2000, p. 25).

Sobre la base de estos antecedentes, en este estudio se privilegia el análisis de trayectorias para revisar la experiencia de los jóvenes egresados de la educación técnica. Como ya se ha indicado, menos que una sucesión de etapas claramente definidas o el desarrollo de un programa preestablecido, en la actualidad la experiencia juvenil y la transición educativo-laboral que estos experimentan están asociadas a nuevos contextos, marcados por el debilitamiento de soportes institucionales como los que conocieron otras generaciones. En este marco, a diferencia de un modelo conceptual clásico, que definía la juventud asociada a un camino relativamente delimitado y fuertemente marcado por una secuencialidad que distinguía la etapa de formación respecto a la vida laboral (y donde quienes transitaban por la formación para el trabajo lo experimentaban con mayor rigor), la actual mirada sobre la realidad juvenil releva la creciente flexibilidad de los límites de este período de vida, la imbricación de los campos de desenvolvimiento de los sujetos y, como consecuencia, la diversidad y complejidad de las trayectorias que experimentan.

\section{ANTECEDENTES METODOLÓGICOS DEL ESTUDIO}

El material empírico sobre el que se organiza este artículo proviene del levantamiento de una investigación en marcha: "Expectativas, proyectos educativo-laborales y trayectorias post-egreso de jóvenes estudiantes secundarios: Un estudio en la Región Metropolitana", que se realiza con el apoyo de FONDECYT $^{8}$. Este estudio, de carácter longitudinal ${ }^{9}$, tiene como objetivo analizar los modelos de trayectoria educativo-laboral que se desprenden de la

\footnotetext{
${ }^{8}$ Fondo Nacional de Desarrollo Científico y Tecnológico de La Comisión Nacional de Investigación Científica y Tecnológica (CONICYT).

${ }^{9}$ Una de las ventajas de los estudios longitudinales es que, a diferencia de estudios retrospectivos, con esta modalidad metodológica es posible analizar el contraste entre las aspiraciones iniciales y los eventuales ajustes posteriores que realizan los individuos, en función de su propia experiencia.
} 
experiencia de una muestra de jóvenes estudiantes durante el período posterior a su egreso de la enseñanza secundaria.

El estudio consiste en la aplicación de olas sucesivas de encuestas y entrevistas cualitativas a jóvenes que cursaban su último año escolar durante el año 2011 en distintos establecimientos de la Región Metropolitana de Santiago de Chile. Con posterioridad han existido cuatro etapas sucesivas de levantamiento de información, entre los años 2012 y 2015, para recoger antecedentes sobre la situación post-egreso de la muestra. La última ola investigativa corresponde al año 2016.

En la primera fase del estudio se implementó una encuesta dirigida a una muestra de estudiantes del último año de enseñanza secundaria pertenecientes a diversos tipos de establecimientos educacionales. En la construcción de esta muestra se consideró la dependencia administrativa de los centros seleccionados y la modalidad de estudios de los jóvenes —modalidad científico humanista $(\mathrm{EMCH}$, de orientación académica) y técnico profesional (EMTP)—. El trabajo de campo para esta primera parte contempló la aplicación de un instrumento estructurado a 1888 estudiantes de $4^{\circ}$ año medio en 69 establecimientos educativos $^{10}$.

En este artículo se recogen antecedentes de esta primera fase de indagación, que sintetizan información comparada acerca de las expectativas y proyectos de futuro de los jóvenes de ambas modalidades formativas. En las encuestas individuales posteriores se recoge la experiencia de los jóvenes una vez egresados de la enseñanza secundaria, y se levanta información acerca del

\footnotetext{
${ }^{10}$ Los criterios de selección de la muestra consideraron como unidad primaria de muestreo los establecimientos educativos. Estos fueron seleccionados de manera aleatoria según su dependencia administrativa (pública, privada con financiamiento estatal o privada) y modalidad de estudio. Adicionalmente, en cada una de las unidades se seleccionó a uno o dos grupos curso del nivel de 4으 medio (último año de secundaria en el sistema chileno) para la aplicación del instrumento. El cuestionario, que incluyó preguntas destinadas a la caracterización general de los estudiantes, su experiencia educativa, intereses personales y consumo cultural, aspiraciones y proyectos de futuro, entre otras dimensiones, fue de carácter auto-aplicado y con la presencia de un encuestador en la sala de clases, quien supervisó el desarrollo del proceso. Las olas siguientes consistieron en encuestas domiciliarias (o lugares de estudio); en los casos en que no fue posible esta modalidad, se aplicó una encuesta telefónica a los jóvenes individualizados.
} 
tipo de trayectoria que estos realizan durante los años siguientes y el grado de cumplimiento de las expectativas originalmente trazadas. Los antecedentes recogidos en la indagación fueron complementados con material empírico de tipo cualitativo, obtenido a través de entrevistas individuales y grupos de discusión con jóvenes incluidos en la muestra ${ }^{11}$.

Los datos recogidos mediante encuestas que se presentan en este artículo han sido sometidos a un análisis estadístico simple, con fines propiamente descriptivos, y aplicado sobre los indicadores relevados en la indagación. Este proceso fue realizado para describir el comportamiento de tales indicadores en la muestra del estudio, identificar la concentración y desconcentración de datos, e identificar la cantidad de casos agrupados para cada categoría de respuesta ${ }^{12}$.

\section{TRAYECTORIAS EDUCATIVO-LABORALES DE JÓVENES EGRESADOS DE LA ENSEÑANZA TÉCNICO PROFESIONAL}

La presentación de los resultados del estudio permite revisar ciertos ámbitos relevantes de la experiencia educativo-laboral, enfatizando un ejercicio analítico de la experiencia de los jóvenes que realizaron estudios técnicos en el nivel de su enseñanza secundaria. Aunque algunos de los antecedentes se presentan en comparación con la situación de sus pares de formación académica o de educación científico humanista, el foco está centrado en caracterizar los antecedentes que remiten al subgrupo de egresados de la educación técnica y su

\footnotetext{
${ }^{11}$ La cuarta etapa de medición en este estudio alcanzó una cobertura de $42,4 \%$ de la muestra inicial, tasa de respuestas que se sitúa en un margen razonable para estudios de estas características. Es lo que se observa (aunque con un tratamiento metodológico y condiciones de realización diferente), por ejemplo, en los estudios "Socioeconomic disadvantage and access to higher education", realizado en cuatro distritos de Glasgow entre 1999 y 2004; "Paths on life's way", realizado a lo largo de la década del 90 en British Columbia, Canadá; o el proyecto "Life Patterns", realizado en Victoria, Australia, en igual período de tiempo. Véase Furlong y Cartmel (2005); Andres y Wyn (2010).

${ }^{12}$ El análisis de la información cualitativa, por su parte, estuvo orientado a caracterizar el discurso predominante en los actores en sus respectivos contextos, para luego elaborar una interpretación global de la información. El análisis del material se organizó a partir de los principios básicos de la teoría fundamentada e incluyó procedimientos de codificación temática con el objetivo de observar una eventual distribución social de las perspectivas sobre los temas de interés investigativo. En el artículo se incluyen algunas citas rescatadas del material cualitativo levantado a lo largo del estudio.
} 
particular experiencia en el contexto educativo-laboral de la sociedad chilena en la actualidad.

La presentación se organiza en tres secciones: (a) las perspectivas de futuro predominantes entre los jóvenes antes de su egreso de la enseñanza secundaria; (b) la incidencia de trayectorias educativas post-secundarias en esta cohorte y (c) la experiencia laboral de estos jóvenes, y muy particularmente de quienes ingresan tempranamente al mundo del trabajo:

\section{Perspectivas de futuro de los jóvenes al egreso de la enseñanza media: altas aspiraciones de continuidad de estudios post-secundarios}

En la primera parte de esta investigación se indagó acerca de los planes y proyectos de largo plazo que tenían los jóvenes mientras aún cursaban el último año de su enseñanza secundaria. Para el grueso de la muestra, predominaba ampliamente el interés por cursar estudios de educación superior como paso previo al inicio de la vida laboral. Un poco más del 60\% de los encuestados indicaba su motivación por realizar estudios post-secundarios y solo un 7\% señalaba que al terminar la enseñanza escolar deseaba ingresar al mundo del trabajo.

Esta tendencia, en lo grueso, era compartida por los estudiantes de las dos modalidades formativas existentes en el sistema chileno. Como puede verse en la tabla n. ${ }^{\circ}$, solo un $11 \%$ de los estudiantes de formación técnica profesional señalaba su disposición a ingresar al mundo del trabajo inmediatamente después de su egreso de la secundaria. A diferencia de esto, al igual que sus pares del sistema de educación académica, la mayoría de los estudiantes de esta modalidad manifestaba su interés por continuar estudios terciarios (60\% de la muestra), aunque con una mayor prevalencia de carreras técnicas de nivel superior. Un segmento menor se inclinaba por caracterizar su realidad como indefinida, mientras que un 15\% optaba por una estrategia destinada a la realización de su 
Trayectorias educativo-laborales de jóvenes estudiantes de educación técnica en Chile

práctica profesional $^{13}$ y una combinación posterior de estudio y trabajo al año siguiente de su egreso ${ }^{14}$ :

Tabla n.o 1

Aspiraciones de futuro educativo-laboral en estudiantes de modalidad EMCH y EMTP ${ }^{15}$

\begin{tabular}{|l|c|c|c|}
\hline & $\begin{array}{c}\text { Modalidad } \\
\mathbf{C H}\end{array}$ & $\begin{array}{c}\text { Modalidad } \\
\text { TP }\end{array}$ & Total \\
\hline Trabajará y no estudiará & $\mathbf{2 . 7 \%}$ & $11 \%$ & $\mathbf{7 , 4 \%}$ \\
\hline Terminará una carrera en una Universidad & $\mathbf{7 3 . 7 \%}$ & $\mathbf{3 1 \%}$ & $\mathbf{4 5 . 2 \%}$ \\
\hline Terminará estudios técnicos superiores & $\mathbf{1 2 . 2 \%}$ & $\mathbf{2 9 . 3 \%}$ & $\mathbf{2 1 . 5 \%}$ \\
\hline Realizará una carrera Militar o Policial & $2.7 \%$ & $5.7 \%$ & $4.2 \%$ \\
\hline No estudiará ni trabajará & $0.4 \%$ & $0.2 \%$ & $0.5 \%$ \\
\hline No lo tiene claro & $5.6 \%$ & $8.3 \%$ & $6.9 \%$ \\
\hline Otro (trayectoria combinada TP) & $2.6 \%$ & $14.6 \%$ & $8.6 \%$ \\
\hline Total & $100 \%$ & $100 \%$ & $100 \%$ \\
\hline
\end{tabular}

Esta primera constatación no constituye una sorpresa para el análisis del sistema técnico profesional de los últimos años. Como lo demuestran algunos estudios recientes (Orellana, 2011), la masificación del sistema de educación superior y el debilitamiento de las credenciales educativas de la educación media han incidido de manera consistente en el incremento de las demandas y aspiraciones de todos los sectores sociales, y muy particularmente de aquellos para los que, hasta hace poco, la continuidad de estudios representaba una posibilidad extremadamente restrictiva. La noción de una formación a lo largo de la vida - tempranamente incorporada en el currículum de la formación técnica

\footnotetext{
${ }^{13}$ La práctica profesional corresponde a una actividad curricular en la modalidad técnica profesional. Corresponde a un plan de desempeño individual de los estudiantes en una empresa del rubro de la especialidad cursada. Esta actividad es gestionada y supervisada por los docentes del centro educativo y tiene una duración que varía entre las 450 y 720 horas cronológicas. Por lo general se realiza al egreso de la enseñanza secundaria y permite la obtención del título de técnico de nivel medio a quienes cumplan satisfactoriamente con este proceso.

${ }^{14}$ La categoría “Otro" reúne las respuestas alternativas que definen los propios estudiantes. En este caso, la mayoría se manifiesta por una combinación de actividades de estudio y trabajo que no calzan, necesariamente, con las alternativas propuestas en el cuestionario, aunque, en un sentido estricto responden mayormente a opciones de estudio y postergación del ingreso al mundo del trabajo. Con todo, para fines del análisis, se optó mantenerla como una categoría aparte.

${ }^{15}$ En negrita corresponde a diferencias estadísticamente significativas a partir de aplicación de prueba t, a un nivel de confianza del $95 \%$.
}

Revista Páginas de Educación. Vol. 9, Núm. 2 (2016) ISSN: 1688-5287; e-ISSN: 1688-7468 
secundaria $^{16} \mathrm{e}$, inicialmente, muy resistida por los agentes educativos del sistema- parece ser, en la actualidad, una perspectiva ampliamente extendida entre los jóvenes estudiantes, que orientan sus proyectos educativo-laborales más allá de los límites de la formación de nivel secundario, y una perspectiva de transición temprana a la vida laboral.

Menos que una desvalorización de la formación recibida, para un segmento importante de los jóvenes la educación técnica parece representar una herramienta que les permite hacer frente a los desafíos económicos inmediatos y les da la posibilidad de financiar los estudios futuros. En este contexto, algunos proyectan la realización de estudios en el área de especialización cursada en la secundaria, mientras que otros simplemente la consideran desde una perspectiva instrumental, al poder optar por alternativas educativas distintas a la formación recibida en su educación técnica escolar. En uno y otro caso, la realización de los estudios técnicos secundarios no representa un punto de quiebre que anuncia el paso de una etapa de vida a otra (la transición educación-trabajo), sino más bien un puente que permite la consolidación de un proyecto de vida personal en el mediano o largo plazo ${ }^{17}$. Esta perspectiva es ampliamente compartida por estudiantes de diversas especialidades del sistema $\mathrm{y}$, en muchos casos, representa un modelo discursivo que supera las propias expectativas de los docentes a cargo de la preparación de los jóvenes.

\footnotetext{
${ }^{16}$ En 1998 se implementó en Chile una reforma curricular de la educación técnica de nivel secundario, que enfatizó en la necesidad de desarrollar una formación que permitiese a los egresados desenvolverse en un sector del mundo productivo, a diferencia de un modelo tradicional de formación destinado al desempeño en un puesto de trabajo. Lo anterior implicaba la apertura de alternativas educativas y la postergación de la toma de decisiones sobre orientación vocacional de los estudiantes, favorecía el acceso a los aprendizajes fundamentales y limitaba las salidas tempranas al mundo del trabajo (Miranda, 2005).

${ }^{17}$ Este argumento aparece de manera reiterada en las entrevistas a los estudiantes y egresados de este sistema. Así lo señalaba una estudiante en un grupo de discusión realizado en el marco de este estudio: "yo estudié esta especialidad principalmente porque es un tema seguro si uno quiere salir a lo laboral. Ahí yo tengo algo seguro y algo estable, y puedo salir con algo que no tiene el (estudiante) $\mathrm{CH}$, que termina el liceo y queda sin nada. Yo pretendo hacer mi práctica y seguir trabajando, porque recursos no tengo para estudiar ahora... así que pretendo trabajar un año y después poder estudiar lo que me interesa, que es profesora" (Estudiante mujer, especialidad administración, entrevista estudio FONDECYT, 2011).
} 


\section{Ingreso a la educación superior de estudiantes técnicos: participación significativa, aunque trayectorias más complejas}

Un segundo punto relevante del estudio tiene que ver con el tránsito post-egreso de la enseñanza secundaria de los jóvenes de la muestra. Al indagar sobre la situación un año después de finalizados los estudios secundarios se ratifica el incremento de la participación de los jóvenes de la modalidad formativa técnicoprofesional en la educación superior, aunque con características particulares al compararse con sus pares de la modalidad de estudios académicos.

Entre los encuestados que estudiaron la modalidad científico humanista, el $70 \%$ ingresó a una carrera de nivel superior, con un alto predominio al interior de carreras de carácter universitario. Solo un poco menos de un $10 \%$ señaló que su actividad principal fue trabajar, mientras que el $16 \%$ de este grupo no estudió ni trabajó durante el año posterior a la finalización de la educación secundaria, sino que prevaleció una actividad de preparación personal para rendir nuevamente el examen de ingreso universitario al año subsiguiente ${ }^{18}$. El interés de continuar estudios es la orientación predominante en este subgrupo, con una baja recurrencia de casos que hayan optado por el trabajo como principal actividad.

A diferencia de lo anterior, entre los jóvenes provenientes de la educación técnica profesional predominó la realización de una actividad laboral durante el primer año posterior a la finalización de la secundaria: un $42 \%$ del total de este grupo. Junto a esto, un poco más del $16 \%$ de los egresados se encontraba realizando su práctica profesional en alguna empresa o centro laboral. Pese a lo anterior, el $31 \%$ ingresó inmediatamente a la educación superior, y es un poco mayor la proporción de casos que lo hicieron en carreras técnicas de nivel superior

\footnotetext{
${ }^{18}$ En Chile, para el ingreso a la gran mayoría de los centros de educación superior universitaria es necesario rendir un examen nacional (la prueba de selección universitaria). Se realiza anualmente y, bajo una ruta lógica, los estudiantes rinden esta prueba el último mes del año de egreso de la enseñanza secundaria (aunque, por cierto, existe la alternativa de rendirla en etapas sucesivas). Las carreras técnicas de nivel superior son impartidas, en su gran mayoría, por centros privados de educación (Institutos Profesionales y Centros de Formación Técnica) y, a diferencia de la mayoría del sistema universitario, no requieren la rendición de la prueba nacional de ingreso a la educación superior. Estas carreras pueden ser de 4 u 8 semestres y sus costos, en promedio, son más bajos que los que se ofrecen en el sistema universitario.
} 
Trayectorias educativo-laborales de jóvenes estudiantes de educación técnica en Chile

respecto a quienes ingresaron a carreras universitarias. La información se sintetiza en la siguiente tabla:

Tabla n.o 2

Situación al primer año de egreso en estudiantes EMCH y EMTP ${ }^{19}$

\begin{tabular}{|l|c|c|}
\hline & Modalidad CH & Modalidad TP \\
\hline Trabaja & $\mathbf{9 . 7 \%}$ & $\mathbf{4 1 . 8 \%}$ \\
\hline Estudia en la Universidad & $\mathbf{5 7 . 3 \%}$ & $\mathbf{1 2 . 4 \%}$ \\
\hline Estudia en un IP o CFT & $13.1 \%$ & $18.3 \%$ \\
\hline Carrera militar o policial & $1.1 \%$ & $0.8 \%$ \\
\hline Transición (prepara estudios) & $15.9 \%$ & $6.6 \%$ \\
\hline Nada & $0.9 \%$ & $3.6 \%$ \\
\hline Otro (transición educativo-laboral) & $\mathbf{2 \%}$ & $16.5 \%$ \\
\hline Total & $100 \%$ & $100 \%$ \\
\hline
\end{tabular}

Al comparar esta situación con las aspiraciones enunciadas un año antes, entre los jóvenes que egresan de la EMTP existe un incremento de casos en que se integran a la vida laboral, y disminuyen en casi 14 puntos aquellos que deseaban cursar estudios superiores. Peso a ello, las diferencias entre la situación deseada y lo que ocurre en la transición inmediata no son muy distintas respecto a los egresados de la modalidad académica y, en conjunto, el desajuste de expectativas no parece ser particularmente llamativo. Esta diferencia, además, en el caso de los jóvenes técnicos, se acorta en los años siguientes.

En efecto, al segundo y tercer año de egreso de la enseñanza media, el porcentaje de jóvenes de educación técnico profesional que se encontraba estudiando una carrera de educación superior se había incrementado en 10 puntos, hasta alcanzar al $43,4 \%$ de la muestra de este sub-grupo (el $25 \%$ en centros de educación técnica de nivel terciario y el 18,4\% restante en universidades). Este incremento denota el desarrollo de una trayectoria más larga en la experiencia de los jóvenes que han estudiado la modalidad técnica.

\footnotetext{
${ }^{19}$ En negrita corresponde a diferencias estadísticamente significativas a partir de aplicación de prueba t, a un nivel de confianza del $95 \%$.

${ }^{20}$ La mayoría de estos casos corresponde a la realización de práctica profesional.
} 
El ingreso diferido a la educación superior (un modelo de trayectoria más extendido) es una característica de los jóvenes egresados de esta modalidad formativa y responde, entre otros factores, a la necesidad de trabajar para apoyar a la familia y/o reunir recursos que puedan ser destinados al financiamiento de los futuros estudios. En contraste con lo anterior, el porcentaje de jóvenes dedicados a una actividad laboral permanece estable durante el período de los tres años siguientes al egreso de la enseñanza secundaria, y alcanza a una cifra cercana al $40 \%$ del total de la muestra de este sub-grupo. Como podrá verse, este segmento es el que presenta mayores dificultades de integración y reconocimiento de sus credenciales formativas en el mercado laboral.

Entre quienes estudiaron a lo largo de este período, la correspondencia entre el sector productivo de la especialidad estudiada en la enseñanza secundaria y la opción posterior en la educación superior se expresaba de manera heterogénea: existían mayores coincidencias en las carreras asociadas a las áreas de tecnología tales como metalmecánica, electricidad y electrónica (donde, además, predomina una mayor proporción de estudiantes hombres), mientras que la disociación entre formación inicial y elección de estudios superiores era más extendida en áreas de administración y comercio (que presenta una mayor proporción de estudiantes mujeres y que concentra cerca del $40 \%$ del total de la matrícula de la EMTP).

Más allá de estas consideraciones, lo que importa recalcar aquí es la evidencia de las transformaciones ocurridas en los últimos años y que inciden en la definición de una modalidad formativa como es la educación técnica. Los resultados de este estudio son coincidentes con algunas investigaciones realizadas a partir de las bases de datos del sistema de educación superior nacional, que señalan que cerca de un $40 \%$ de quienes terminan su enseñanza media se incorpora a algún programa de estudios post-secundario, con una distribución relativamente proporcional entre carreras universitarias y de nivel técnico superior. Los antecedentes también indican que en este segmento de jóvenes el acceso a estudios terciarios es diferido en el tiempo, aunque existe una mayor predominancia de casos de abandono de los estudios, sin contarse con 
antecedentes suficientes respecto a sus causas concretas (Bassi, 2007; Larrañaga et al, 2013).

En síntesis, la evidencia demuestra que la continuidad de estudios postsecundarios es una alternativa abierta para un porcentaje relevante de los egresados de la enseñanza técnica. $Y$ aunque existe un segmento igualmente importante de jóvenes que no pueden o no desean continuar una formación de carácter terciario, de manera evidente la educación técnica de nivel medio ha dejado de ser una estación terminal o una vía segregada que conduce directamente a la vida del trabajo. Por cierto, esta situación ha sido posible gracias a la expansión de un mercado educativo orientado al desarrollo de carreras de nivel técnico y la ampliación de ofertas de financiamiento (becas y créditos) que entrega el Estado para que los jóvenes de menos recursos puedan financiar sus estudios. Pero, ¿qué ocurre con aquellos jóvenes que siguen o deben seguir ese camino?

\section{Trayectorias laborales: experiencias de precariedad y dificultad de consolidación en el trabajo para quienes no continúan estudios superiores}

Como se ha indicado, en este estudio un porcentaje cercano al $40 \%$ de los egresados de la educación técnica no ingresa a la educación superior e inicia su vida laboral una vez finalizada su enseñanza secundaria (situación que se consolida luego de cuatro años de egreso).

La necesidad de trabajar inmediatamente después del egreso de la educación obligatoria es una realidad extendida entre los jóvenes de este sistema formativo y se expresa de manera marcada en las respuestas obtenidas en las distintas mediciones aplicadas a lo largo de la investigación. De hecho, al cuarto año de egreso el $82 \%$ de los jóvenes de la modalidad técnica han realizado alguna actividad laboral remunerada, porcentaje que contrasta con el 44\% reportado en la muestra de jóvenes que egresaron del sistema científico humanista.

Entre quienes trabajan y no estudian durante el año siguiente a la finalización de la enseñanza media, un 49\% señaló que lo hacía para aportar 
dinero para el hogar, mientras que un $40 \%$ señaló que lo hacía para reunir dinero que permitiese financiar sus futuros estudios. De este grupo, un 38\% señaló que no pudo estudiar inmediatamente después del egreso de la enseñanza media por los problemas económicos de su familia.

Sin considerar a quienes se mantienen inactivos durante este período (que en la muestra del estudio representan un segmento extremadamente marginal ${ }^{21}$ ), dos años después del egreso de la enseñanza media el cuadro de experiencia laboral presenta una realidad heterogénea. Tal como se muestra en la tabla $\mathrm{n} . \stackrel{0}{3}$, existe un segmento de jóvenes que cursaron sus estudios en la modalidad de educación técnica y que continúan una actividad laboral (fundamentalmente en un empleo de carácter dependiente) en el área de la especialidad estudiada: cerca de un tercio de los encuestados pertenecientes a este grupo señala esta situación. Sin embargo, el grueso de los jóvenes, durante el período indicado, se desempeña en actividades laborales de servicios que no requieren una formación especializada como la que se define en el currículum técnico profesional de nivel secundario en Chile.

El $45 \%$ de los egresados de la educación técnica que continúa una actividad laboral lo hace en actividades de servicios de atención a clientes, cajeros u otras actividades comerciales, tareas que en escasas ocasiones están a asociadas a trabajos cualificados y que no requieren el manejo de competencias como las que se definen curricularmente para la formación de las distintas especialidades que se imparten en la educación técnica profesional. Por lo general, se trata de actividades de tiempo parcial y que no posibilitan el desarrollo de una carrera laboral en el lugar de trabajo al tratarse, en la gran mayoría de los casos, de empleos esporádicos.

Tabla n. 03

\footnotetext{
${ }^{21}$ De acuerdo al Instituto Nacional de la Juventud, la incidencia del grupo que no trabaja ni estudia en Chile corresponde al 11\% de la población entre 15 y 29 años, con una predominancia del $87 \%$ de mujeres (INJUV, 2014). Un estudio de seguimiento, como el estudio de trayectorias que aquí se presenta, no es la mejor alternativa para dimensionar el problema debido, entre otras razones, a que un porcentaje importante de los jóvenes en esta situación son desertores tempranos de la enseñanza secundaria.
} 
Trayectorias educativo-laborales de jóvenes estudiantes de educación técnica en Chile

Actividad laboral desarrollada: jóvenes de la muestra que declaran realizar un trabajo como actividad principal durante el segundo año de egreso, por modalidad de estudios

\begin{tabular}{|l|c|c|}
\hline \multicolumn{1}{|c|}{$\begin{array}{c}\text { Por favor, señala en qué trabajas/te } \\
\text { recibiendo una remuneración }\end{array}$} & Estudiantes TP & Estudiantes no TP \\
\hline $\begin{array}{l}\text { En una empresa en la especialidad que } \\
\text { estudiaste (estudiantes de EMTP) }\end{array}$ & $28,4 \%$ & \\
\hline $\begin{array}{l}\text { De manera independiente en la especialidad } \\
\text { que estudiaste (estudiantes de EMTP) }\end{array}$ & $3 \%$ & - \\
\hline $\begin{array}{l}\text { De vendedor(a), cajero(a) u otra actividad en } \\
\text { un local comercial }\end{array}$ & $23,1 \%$ & $34,1 \%$ \\
\hline Como obrero en una empresa o construcción & $5,9 \%$ & $11,4 \%$ \\
\hline $\begin{array}{l}\text { Como empleado en una empresa (junior, } \\
\text { atención de clientes, etc.) }\end{array}$ & $21,9 \%$ & $18,2 \%$ \\
\hline En actividades agrícolas & $0,6 \%$ & $0 \%$ \\
\hline $\begin{array}{l}\text { En actividades de servicio como peluquería, } \\
\text { cuidado de niños, mozo, etc. }\end{array}$ & $1,2 \%$ & $9,1 \%$ \\
\hline Desarrollo de un negocio personal & $1,8 \%$ & $2,3 \%$ \\
\hline $\begin{array}{l}\text { Trabajo familiar (en microempresa, local } \\
\text { comercial, etc.) }\end{array}$ & $100 \%$ & $22,7 \%$ \\
\hline Otra actividad & $1,8 \%$ & \\
\hline Total & $12,4 \%$ & \\
\hline
\end{tabular}

Los antecedentes indican que el tipo de trabajo al que acceden los jóvenes egresados de la enseñanza media técnico profesional refleja las precarias oportunidades existentes en el ámbito laboral para quienes solo disponen de su licencia secundaria.

Aunque en general las respuestas a los cuestionarios reportan que se trata de trabajos formales y en jornadas de horario extendido, los requerimientos para el desempeño en los puestos de trabajo estarían particularmente disociados de una perspectiva de formación técnica exigente o la cualificación alcanzada con una certificación de técnico medio. A diferencia de lo anterior, estos jóvenes más bien 
prosiguen trayectorias laborales similares a las recorridas por los jóvenes sin calificación profesional, como ocurre con los egresados de la modalidad académica y que no continúan estudios posteriores o aquellos que no finalizan estudios secundarios.

Los testimonios recogidos en entrevistas a quienes se encuentran esta situación, aunque evidencian el desajuste de expectativas en función de la formación recibida, no necesariamente dan cuenta de un cuadro de abandono de proyectos de futuro o inexistencia de construcción de sentido de las actividades realizadas. Desde este punto de vista, la situación de trabajo, más allá de la desvinculación con un modelo formativo, también puede ser leída como un proceso de acumulación de experiencias con perspectiva de alcanzar metas más ambiciosas en etapas posteriores (esta situación es destacada, para el caso argentino, en Jacinto, Wolf, Bessega y Longo, 2007).

Junto a lo anterior, no debe obviarse el hecho de que algunas especialidades formativas ligadas a la actividad industrial, fundamentalmente, también presentan características de mayor adecuación al mercado del trabajo y permiten que un número significativo de jóvenes puedan transitar por un camino de mayor articulación, al otorgarle un sentido efectivo a la formación recibida en el nivel de la enseñanza secundaria ${ }^{22}$. Sin embargo, se trata de un segmento reducido, al considerar el grueso de la matrícula de este sistema formativo, que concentra más del $70 \%$ de sus estudiantes en especialidades administrativas y de servicios.

De este modo, entre los jóvenes que no realizan estudios en los años sucesivos, y muy particularmente entre aquellos que pertenecen a los sectores modernos de la economía, la experiencia laboral tiende a estar mayormente disociada del área formativa de base, y predomina una secuencia de empleos en

\footnotetext{
${ }^{22}$ Larrañaga et al (2013) indican que esta situación también se refleja en el nivel de salarios: las mejores remuneraciones se sitúan en especialidades ligadas al sector industrial (construcciones metálicas, electricidad, mecánica automotriz, química o electrónica), mientras que las remuneraciones más bajas se encuentran en actividades de servicios y administración. Se verifica, de manera complementaria, una segmentación de género toda vez que existe una relación directa de la distribución de hombres y mujeres con estos sectores de especialidades técnico-profesionales.
} 
el sector servicios y en tareas que, en sentido estricto, no requerirían una preparación en una profundidad y amplitud como la que se intenta desde el modelo de la EMTP $^{23}$. Este último punto es el más relevante para un debate acerca de las políticas que buscan articular educación y trabajo, al establecer interrogantes consistentes acerca de uno de los fundamentos principales de este modelo formativo y relevar la importancia creciente que adquiere la certificación de estudios superiores en el mercado laboral ${ }^{24}$.

Finalmente, es importante indicar que la experiencia de trabajo es también una situación recurrente entre los egresados de la enseñanza técnica que continúan estudios $^{25}$. En este caso, la experiencia laboral constituye un soporte a su actividad principal, lo que permite el financiamiento de una carrera o contribuye a los gastos asociados a la vida estudiantil. Del total de jóvenes que desarrollan una trayectoria continua de estudios durante los dos años siguientes a su egreso, la mitad de ellos lo hace combinando actividades de estudio y trabajo. Entre los estudiantes de la modalidad científico humanista, esta situación se verifica en un poco menos de un cuarto de quienes se insertan en la educación superior.

Aunque entre los jóvenes predomina una actitud positiva frente a esta situación - que ratifica la importancia de la formación para el trabajo recibida en la enseñanza secundaria como una herramienta para hacer frente al futuro-, los reportes de tipo cualitativo acerca de la experiencia de combinar actividades indican, muchas veces, la complejidad de alcanzar la articulación, particularmente

\footnotetext{
${ }^{23}$ Como una forma de contraste, el testimonio de un joven que continúa estudios de educación superior evidencia las dificultades de inserción laboral para quienes cuentan solo con la certificación de técnico medio: “¿En qué puedo trabajar? Se ve muy limitado y conozco gente que trabaja y es muy limitado lo que puede hacer o lo que le piden. A veces hay que hacer mucho esfuerzo para conseguir algo que a veces es muy inferior a lo que uno tenía pensado, o lo que pensaba que podía hacer con lo que estudió en el liceo... por eso yo quiero seguir estudiando, además me creo muy capaz de seguir estudiando" (Estudiante hombre, especialidad sector servicios, entrevista estudio FONDECYT, 2012).

${ }^{24}$ El desajuste entre la oferta educativa respecto a la demanda laboral es un tema de larga data y que, en el caso de la educación técnica, ya fue destacado en otro contexto histórico de desarrollo. Véase Wiñar (1981).

${ }^{25}$ Esta situación también es observada en estudios realizados en Argentina. En este país, además de evidenciarse un alto porcentaje de jóvenes que continúan estudios superiores (63\% de una muestra censada en 2009), se encontró que la mitad lo hacía bajo la modalidad de combinación estudio y trabajo (Sosa, 2015).
} 
en aquellos empleos de mayor formalidad y vinculación al perfil profesional de los egresados $^{26}$. De este modo, la exigencia asociada al desarrollo de una carrera profesional de nivel superior orientaría hacia la elección de empleos de menor calificación y mayor flexibilidad, lo que limita el potencial laboral de estos jóvenes y profundiza la brecha entre dos procesos, estudio y trabajo, que en el corto y mediano plazo no terminan de encontrarse.

\section{A MANERA DE SÍNTESIS}

En lo grueso, los resultados del estudio que se presentan aquí:

(a) ratifican el incremento de la participación de los jóvenes de la modalidad técnico profesional en la educación superior, y evidencia que ya no se trata de un modelo formativo de ingreso temprano al mundo del trabajo;

(b) aunque los estudiantes priorizan estudiar en carreras técnicas ofrecidas en centros no universitarios de formación superior, un porcentaje no menor también se incorpora a carreras universitarias en áreas disímiles a las de su formación inicial. Más allá de esto, la experiencia de combinar estudios y trabajo es una situación recurrente en un segmento importante de estos jóvenes, lo que ratifica un sentido instrumental de la formación para el trabajo recibida en la educación secundaria, necesaria para hacer frente a las necesidades económicas de la gran mayoría de quienes egresan de este sistema educativo; (c) la correspondencia entre el sector productivo de la especialidad estudiada en la enseñanza secundaria y la opción posterior en la educación superior es heterogénea, y resulta mucho más evidente en las áreas de tecnología. A diferencia de lo anterior,

\footnotetext{
${ }^{26}$ El testimonio de un joven egresado de educación técnica y que continúa estudios en su especialidad refleja esta situación: "Yo estudié la especialidad de telecomunicaciones y ahora me encuentro en el quinto semestre de la carrera de ingeniería en informática. Hasta el año pasado estaba trabajando, pero tuve dejarlo porque era difícil trabajar y estudiar al mismo tiempo. Mis viejos ahora me están financiando los estudios. Trabajaba en el aeropuerto en la parte de soporte informático, pero resulta que donde estaba estudiando nos dan muchos trabajos e investigaciones, además de asistir a clases, lo que me complicaba. Además, el trabajo se prolongaba y a veces no podía llegar a clases al día siguiente... finalmente tuve que optar, porque no podía hacer las dos cosas" (Estudiante hombre, especialidad informática, entrevista estudio FONDECYT, 2014).
} 
la disociación entre formación inicial y elección de estudios superiores es mayor en áreas de administración y comercio, las que concentran cerca del $40 \%$ del total de la matrícula técnica de nivel secundario. Desde este punto de vista la especialidad cursada en secundaria no opera, necesariamente, como una camisa de fuerza para las trayectorias posteriores de estos jóvenes, y es posible hipotetizar que aquellos que continúan en el área de formación recibida en la secundaria lo hacen respondiendo más a un interés vocacional que a un determinismo externo del que no pudiesen desprenderse; (d) finalmente, entre los jóvenes que no realizan estudios en los años sucesivos, la experiencia laboral tiende a estar mayormente disociada del área formativa de base, y predomina una secuencia de empleos en el sector servicios y en tareas que, en sentido estricto, no requerirían una preparación de como la que se intenta desde el modelo de la EMTP. Este último punto es el más crítico y, por cierto, evidencia el desajuste que existiría entre la certificación de estudios secundarios de nivel técnico y las posibilidades efectivas que ofrece el mercado de trabajo a quienes portan estas credenciales.

Desde una perspectiva general, los antecedentes que aquí se presentan, menos que cuestionar la relevancia de un sistema de educación diferenciado en la enseñanza secundaria, obligan a considerarlo en su actual complejidad. La experiencia de los estudiantes que egresan de este sistema evidencia las múltiples trayectorias que estos pueden seguir en el continuo educación-trabajo, y las dificultades ciertas que implica enfrentar tempranamente un mercado laboral que, muchas veces, no resulta funcional a los diseños curriculares que se construyen desde el ámbito educativo.

Lo que sugiere la experiencia de los jóvenes participantes de este estudio refuerza una perspectiva de la educación técnica que ha estado presente desde hace varios años en el debate internacional, y que critica la persistencia de un modelo educativo independiente y paralelo al sistema educativo formal, orientado 
únicamente a la inserción temprana al mundo del trabajo ${ }^{27}$. A diferencia de esto, considerar a la educación técnica como un sistema integrado a un modelo mayor, esto es, un sistema de formación permanente que evite la segregación de vías formativas y la distinción de un modelo de educación superior exclusivamente de corte académico, parece ser una estrategia que no solo se corresponde con las transformaciones socioproductivas de las últimas décadas, sino también con las expectativas y las propias estrategias de futuro que construyen los jóvenes.

Este hecho orienta a trabajar para reforzar una formación mayormente enfocada al desarrollo de capacidades personales que entregue herramientas para el desarrollo autónomo, al otorgarle mayor peso al desarrollo del conocimiento tecnológico y el fortalecimiento de competencias transversales, menos asociadas a una especialidad formativa en particular y mayormente relacionadas con las transformaciones y exigencias cotidianas del mundo actual.

La integración curricular de la formación vocacional con las modalidades tradicionalmente académicas en el nivel secundario (donde esta última también requiere una vinculación a los temas de trabajo y competencias de empleabilidad), así como la construcción de puentes que faciliten recorridos hacia el nivel de educación superior, incluida la de tipo técnico profesional, parecen ser alternativas de trayectorias necesarias, que la experiencia de los jóvenes ha venido ratificando en los últimos años.

\footnotetext{
${ }^{27}$ El discurso que concibe la educación técnica como un modelo formativo destinado a la preparación e inserción temprana en el mundo del trabajo de jóvenes pobres, y su funcionalidad a los requerimientos de la productividad, sigue siendo predominante en diversos ámbitos de la discusión, muy particularmente en los organismos económicos internacionales. Véase por ejemplo BID (2013); Listerri, Gligo, Homs y Ruíz-Devesa (2014).
} 


\section{REFERENCIAS}

Andres, L. y Wyn, J. (2010). The making of a generation: the children of the 1970s in adulthood. Toronto: University of Toronto Press.

Bassi, M. (2007). Educación Técnica en Chile: Conclusiones de una encuesta sobre inserción laboral de los jóvenes. Santiago: BID.

BID. (2013). Una buena educación técnica empieza por... Aportes, 21, 1-4. Recuperado de: http://idbdocs.iadb.org/wsdocs/getdocument.aspx?docnum=37951456

Biggart, A., Furlong, A. y Cartmel, F. (2008). Biografías de elección y linealidad transicional: nueva conceptualización de las transiciones de la juventud moderna. Bendit, R., Hanh, M. y Miranda, A. (Comps.). Los jóvenes y el futuro: procesos de inclusión social y patrones de vulnerabilidad en un mundo globalizado (pp. 49-72). Buenos Aires: Prometeo.

CEPAL. (1997). La brecha de la equidad: América Latina, el Caribe y la Cumbre Social. Santiago: CEPAL.

CEPAL-UNESCO. (1992). Educación y conocimiento: Eje de la transformación productiva con equidad. Santiago: Tarea.

Cieslik, M. y Simson, D. (2013). Key concepts in youth studies. London: SAGE.

Dávila, O., Ghiardo, F. y Medrano, C. (2005). Los desheredados: Trayectoria de vida y nuevas condiciones juveniles. Valparaíso: CIDPA Ediciones.

Di Gropello, E. (Ed.). (2006). Meeting the challenges of secundary education in Latin America and East Asia. Washington: The World Bank.

Educación 2020 (2016). 15 propuestas para cambiar la historia de la Educación Técnica. Recuperado de: http://www.educacion2020.cl/noticia/las-15-propuestas-de-lasociedad-civil-para-mejorar-la-educacion-tecnica

Espinoza, O., Catillo, D. y Traslaviña, P. (2011). La implementación de la reforma curricular en la Educación Media Técnico Profesional: evaluación y proyecciones. Santiago: CIE, PIIE, Universidad UCINF.

Evans, K. (2002) Talking control of their lives?: Agency in young adult transitions in England and the New Germany. Journal of Youth Studies, 5 (3), 245-270.

Farías, M. y Carrasco, R. (2012). Diferencias en resultados académicos entre la educación media técnico profesional y humanista científica en Chile. Calidad en la Educación, 36, 88-121. 
Trayectorias educativo-laborales de jóvenes estudiantes de educación técnica en Chile

Fridman, V. y Otero, A. (2015). De estudiantes a trabajadores. Miranda, A. (Comp.) Sociología de la educación y transición al mundo del trabajo (pp. 169-198). Buenos Aires: Teseo-FLACSO.

Furlong, A. (2011). El cambio social y las transiciones de la educación al mundo laboral en Asociación Internacional de Ciudades Educadoras. Monográfico. Ciudad, Juventud y Educación. Recuperado de: http://www.edcities.org/wpcontent/uploads/2011/07/Monografico-Ciudad-Juventud-y-educacion.pdf

Furlong, A. (2009). Revisiting transitional metaphors: reproducing social inequalities under the conditions of late modernity. Journal of Education and Work, 22 (5), 343-353.

Furlong, A. y Cartmel, F. (2005). Graduates from disadvantaged families: Early labour market experiences. London: Joseph Rowntree Foundation.

Heinz, W. (2009). Youth transitions in age of uncertainty. Furlong, A. (Ed.). Handbook of youth and young adulthood; new perspectives and agendas. London: Routledge.

Heinz, W. y Krüger, H. (2001). Life course: innovations and challenges for social research. Current Sociology, 49 (2), 29-45.

INJUV. (2014). Tendencias: Generación NINI, jóvenes que ni estudian ni trabajan. $R T$ Blog, 10. Recuperado de: http://www.injuv.gob.cl/portal/rt-blog/numero-10septiembre-2014/tendencias-generacion-nini-jovenes-que-ni-estudian-ni-trabajan/

Jacinto, C. (Comp.). (2010). La construcción social de las trayectorias laborales de jóvenes: Políticas, instituciones, dispositivos y subjetividades. Buenos Aires: Teseo IDES.

Jacinto, C., Wolf, M., Bessega, C. y Longo, M.E. (2007). Jóvenes, precariedades y sentidos del trabajo. Presentado en el $7^{\circ}$ Congreso Nacional de Estudios del Trabajo de ASET, Buenos Aires, Argentina. Recuperado de: http://www.aset.org.ar/congresos/7/02007.pdf

Larrañaga, O., Cabezas, G. y Dusaillant, F. (2013). Educación técnico profesional: trayectoria educacional e inserción laboral de una cohorte de alumnos. Santiago: PNUD. Recuperado de: http://www.undp.org/content/dam/chile/docs/pobreza/undp cl pobreza etp 2013.p df

Leccardi, C. y Ruspini, E. (Eds.). (2006). A new youth?: Young people, generations and family life. Farnham: Ashgate. 
Trayectorias educativo-laborales de jóvenes estudiantes de educación técnica en Chile

Listerri, J.J., Gligo, N., Homs, O. y Ruíz-Devesa, D. (2014). Educación técnica y formación profesional en América Latina: el reto de la productividad. CAF - Banco de Desarrollo de América Latina. Serie Políticas Públicas y Transformación Productiva, 13. Recuperado de: http://publicaciones.caf.com/media/38365/educacion tecnica formacion profesion al.pdf

Maclean, R. y Pavlova, M. (2013). Vocationalization of secondary and higher education: pathways to the world of work. UNESCO-UNEVOC. Revisiting global trends in TVET: Reflections on theory and practice (pp. 40-85). Recuperado de:

http://www.unevoc.unesco.org/fileadmin/up/2013 epub revisiting global trends in tvet bo ok.pdf

Machado Pais, J. (2000). Las transiciones y culturas de la juventud: formas y escenificaciones. Revista Internacional de Ciencias Sociales, 164, 89-101.

Mejer, F. (2013). Estado de situación de los sistemas de información de la Educación Técnica y Profesional (EFTP) en 12 países de América Latina y el Caribe.

Santiago: UNESCO. Recuperado de: http://www.unesco.org/new/fileadmin/MULTIMEDIA/FIELD/Santiago/pdf/ESTADO SITUACI ON TVET-esp.pdf

Ministerio de Educación. (2013). Bases curriculares. Formación diferenciada técnico profesional. Especialidades y perfiles de esgreso. Recuperado de: http://www.tecnicoprofesional.mineduc.cl/wp-content/uploads/2016/03/Basesformacion-Descripci\%C3\%B3n-de-las-Especialidades.pdf

Ministerio de Educación. (2009). Informe Ejecutivo. Bases para una política de formación técnico profesional en Chile. Recuperado de: http://portales.mineduc.cl/usuarios/1234/File/Formacion\%20Tecnica/Informe base sFormTecnProf Chile2009.pdf

Miranda, M. (2005). Transformaciones de la Educación Media Técnico Profesional. Cox, C. (Ed.) Políticas educacionales en el cambio de siglo: La reforma del sistema escolar en Chile (pp. 375-417). Santiago: Universitaria.

Miranda, A. y Otero, A. (2005). Diversidad y desigualdad en los tránsitos de los egresados de la escuela secundaria. Revista Mexicana de Investigación Educativa, 10 (25), 393-417.

Orellana, V. (2011). Nuevos estudiantes y tendencias emergentes en la Educación Superior: una mirada al Chile de mañana. Jiménez, M. y Lagos, F. (Eds.). Nueva 
Trayectorias educativo-laborales de jóvenes estudiantes de educación técnica en Chile

geografía de la educación superior y de los estudiantes: Una cartografía del sistema chileno, su actual alumnado y sus principales tendencias pp. 80-142. Santiago: Universidad San Sebastián.

Sarojini, C. (2014). Aspirations, Education and Social Justice: Applying Sen and Bourdieu. London: Bloomsbury.

Sepúlveda, L., Ugalde, P. y Campos, F. (2011). La enseñanza media técnico profesional en Chile: orientaciones actuales desde la perspectiva de sus actores. Centro de Estudios MINEDUC. Evidencias para políticas públicas en Educación: Selección de Investigaciones Tercer Concurso FONIDE. Tomo I (pp. 15-52). Santiago: MINEDUC.

Sevilla, P. (2012). Educación Técnica Profesional en Chile: Antecedentes y Claves de Diagnóstico. Recuperado de:

http://www.mineduc.cl/usuarios/mineduc/doc/201204241130130.DiagnOsticoEducaciOnTP CentrodeEstudiosMINEDUC.pdf

Sevilla, P., Farías, M. y Sepúlveda, L. (2013). Una nueva perspectiva para la educación técnica: condiciones y propuestas asociadas. Cuaderno de Educación, 56, 1-4. Recuperado de: http://mailing.uahurtado.cl/cuaderno educacion 56/pdf/actualidad 56.pdf

SIES-MINEDUC. (2014). Transición de Educación media a educación superior. Experiencia de cohorte de egreso 2006. Recuperado de: http://www.mifuturo.cl/images/Estudios/Estudios SIES DIVESUP/transicion cohorte 2006 a ed superior 2014.pdf

Sosa, M. L. (2015). ¿Los jóvenes egresados de escuelas técnicas se insertan mejor en el mercado laboral que el resto de los graduados de secundario en la Argentina actual? Presentado en el $12^{\circ}$ Congreso Nacional de Estudios del trabajo de ASET, Buenos Aires, Argentina.

Staff, J. y Mortimer, J. (2003). Diverse Transitions from School to Work. Work and Occupations, 30, 361-369.

Stern, D. (2009). Expanding policy options for educating teenagers. America's High Schools, 19 (1), 211-239.

UNESCO. (2012). Situación Educativa de América Latina y el Caribe: Hacia la educación de calidad para todos al 2015. Recuperado de: http://www.unesco.org/new/fileadmin/MULTIMEDIA/FIELD/Santiago/images/SITIEDespanol.pdf 
Trayectorias educativo-laborales de jóvenes estudiantes de educación técnica en Chile

UNESCO. (2016). Proyecto de estrategia para la enseñanza y formación técnica y profesional (EFTP) (2016-2021). Recuperado de:

http://unesdoc.unesco.org/images/0024/002438/243804s.pdf

Vera, A. y Castioni, R. (2010). Los Jóvenes en Latinoamérica. La transición escuelatrabajo como objeto de políticas públicas. Boletín Técnico SENAC, 36 (2), 5-17.

Wallenborn, M. y Heyneman, S. (2009). Should vocational education be part of secondary education? Journal of Educational Change, 10, 405-413.

Willis, P. (2003). Foot Soldiers of Modernity: The dialectics of cultural consumption and the 21st century school. Harvard Educational Review, 73 (3), 390-415.

Wiñar, D. (1981). Educación Técnica y estructura social en América Latina. UNESCO, CEPAL, PNUD. Recuperado de: http://repositorio.cepal.org/bitstream/handle/11362/28572/S8100592 es.pdf;sequence=1

Woodman, D. y Wyn, J. (2011). Youth research in a changing world. Beadle, S., Holdsworth, R. y Wyn, J. (Eds.). For we are Young and...?: Young people in a time of uncertainty (pp. 5-28). Melbourne: Melbourne University Press.

Wyn, J. y Dwyer, P. (2000). Nuevas pautas en la transición de la juventud en la educación. Revista Internacional de Ciencias Sociales, 164, 17-29. 